Trascender, Contabilidad y Gestión. Vol. 7, Núm. 19 (enero - abril del 2022).

Universidad de Sonora. Departamento de Contabilidad. México.

ISSN: 2448-6388. Reserva de Derechos 04-2015-04172070800-203.

\title{
Estudio de caso del programa de inclusión laboral a personas con discapacidad en el sureste mexicano
}

\section{Case study of a labor inclusion program for people with disabilities in the Mexican southeast}

\author{
Guadalupe Zimbrón Pérez ${ }^{1}$; Ruth Noemí Ojeda López ${ }^{2}$
}

Recibido: 14 de agosto de 2021.

Aceptado: 12 de octubre de 2021.

DOI: https://doi.org/10.36791/tcg.v7i19.132

JEL: J14. Economía de los discapacitados y código.

J15. Economía de las minorías y de las razas; No discriminación laboral.

\section{Resumen}

El objetivo de este artículo es analizar por qué la inclusión laboral a Personas con Discapacidad (PCD) ha ido en decremento durante el periodo 2013-2018 en el sureste mexicano, a pesar de que en el país se cuenta con diferentes legislaciones, mecanismos y programas (nacionales y estatales) enfocados a la inclusión laboral a PCD, la metodología fue a través de estudio de caso a un programa de rehabilitación e inclusión laboral, por medio de entrevistas, observación participante, revisión documental de diferentes fuentes de información y políticas públicas de reciente creación. Como resultado, para la mayoría de los empresarios participantes del programa, la inclusión laboral a PCD fue una experiencia positiva, se concluye que un programa de inclusión laboral sólo es el inicio hacia la inclusión de PCD en el ámbito laboral, pero no es suficiente. La inclusión necesita que las autoridades, sociedad en general,

\footnotetext{
${ }^{1}$ Guadalupe Zimbrón Pérez. Maestra en Dirección Estratégica de Empresas Familiares. Investigador independiente del Doctorado en Ciencias Sociales de la Universidad Autónoma de Yucatán, Mérida, Yucatán, México. Correo: lupi_zp@hotmail.com. ORCID: https://orcid.org/00000002-5710-3815.

${ }^{2}$ Ruth Noemí Ojeda López. Doctora en Ciencias de la Administración. Profesor investigador de la Universidad Autónoma de Yucatán, Mérida, Yucatán, México. Certificada por la Asociación Nacional de Facultades y Escuelas de Contaduría y Administración (ANFECA). Miembro del Sistema Nacional de Investigadores nivel 1. Correo: ruth.ojeda@correo.uady.mx. ORCID: https://orcid.org/0000-0002-120X.
} 
PCD y academia se involucren e informen del tema. Todos somos responsables en nuestras acciones diarias de hacer real no sólo la inclusión laboral, sino también la social.

Palabras clave: discapacidad, estigma, inclusión laboral, discriminación.

\section{Abstract}

The aim of this article is to understand the reason of the labor inclusion of PWD has decreased during the 2013-2018 period in the Mexican southeast, despite in the country has different laws, mechanisms, and programs (national and state) focused on the labor inclusion of $P W D$, the methodology was through a case study of a rehabilitation and labor inclusion program, through interviews, participant observation, documentary review of different sources of information and recently created public policies. As a result, for most participants of labor inclusion program, labor inclusion of PWD was a positive experience. As a conclusion, labor inclusion program is just the beginning towards the inclusion of PWD in the workplace, but it is not enough. The inclusion requires that the authorities, society in general, PWD and academia must be involved and informed. We all are all responsible with our daily actions to achieve not only labor inclusion, also social.

Keywords: disabilities, stigma, labor inclusion, discrimination.

\section{Introducción}

La Clasificación Internacional del Funcionamiento de la Discapacidad y de la Salud (OMS, 2001) define la discapacidad como un término genérico que abarca deficiencias, limitaciones de la actividad y restricciones a la participación e incluye la interacción entre las personas que padecen alguna enfermedad (por ejemplo, parálisis cerebral, síndrome de Down y depresión) y factores personales y ambientales (por ejemplo, actitudes negativas, transporte $\mathrm{y}$ edificios públicos inaccesibles y un apoyo social limitado).

Por su parte, el Programa Nacional de Trabajo y Capacitación para Personas con Discapacidad (PCD) en México del Gobierno Federal (2010-2012), define inclusión laboral de las PCD como la inclusión de las y los trabajadores con discapacidad para trabajar en igualdad de condiciones que los demás, en un universo abierto donde la sociedad debe facilitar y flexibilizar las oportunidades en un ambiente propicio para el desarrollo emocional, físico e intelectual. Se entiende por inclusión laboral no solamente la incorporación de una persona con discapacidad para cubrir una vacante de acuerdo con su perfil y los requerimientos del puesto de trabajo. El término involucra desde la publicación de las vacantes, los procesos de selección y reclutamiento, la capacitación en y para el trabajo, la rehabilitación laboral, accesibilidad, seguridad y salud, igualdad en la remuneración y otras prestaciones, hasta los mecanismos para asegurar la estabilidad en el empleo, la promoción y el ascenso, entre otros (Gobierno Federal, 2010).

A nivel mundial, según datos de la Organización Mundial de la Salud (OMS, 2011), más de mil millones de personas, un $15 \%$ de la población mundial padece discapacidad en alguna forma. Eso no es todo, pues las tasas de discapacidad están aumentando debido en parte, al envejecimiento de la población y al aumento de la prevalencia de enfermedades crónicas.

En el estado del sureste mexicano donde se realizó el estudio de caso, la tasa de población de PCD es de 65 por cada mil habitantes, mientras que la tasa promedio en el país es de 60, con esta cifra el estado ocupa el 
lugar número 13 con la tasa de población con discapacidad por entidad federativa superando la media nacional (INEGI, 2016).

Una investigación de la Organización para la Cooperación y el Desarrollo Económico (OCDE, 2010) demostró que, en 27 países, las PCD en edad de trabajar, en comparación con las Personas sin Discapacidad (PSD), percibían desventajas significativas en el ámbito laboral y tenían peores oportunidades de empleo. En promedio, su tasa de empleo (44\%) era ligeramente superior a la mitad del de las PSD (75\%). La tasa de inactividad era unas 2,5 veces mayor entre las PSD (49\% y 20\%, respectivamente).

En lo relacionado al tema de actividad económica en México, el Instituto Nacional de Estadística y Geografía (INEGI ,2016) reportó que, de cada 10 PCD, solamente 4 participan en actividades económicas, ello contrasta con lo observado entre las PSD las cuales participan en el mercado laboral cerca de 7 de cada 10. Mientras que la tasa de participación económicamente activa de las PCD en el estado del sureste mexicano es de 35.4, y el 68.0 corresponde a las personas que no tienen ningún tipo de discapacidad, siendo la media de la tasa de participación económicamente activa para las PCD de 39.1 y la media para las PSD de 65.7, esto sitúa al estado en la posición número 26 (por debajo de la media) en referencia a las 32 entidades federativas restantes.

Actualmente no se cuenta con datos exactos de la participación económicamente activa de las PCD en la capital del estado del sureste mexicano, siendo necesario mencionar la diferencia entre los registros censales del país de los años 2000 y del 2010 de las PCD, debido a los cambios que se realizaron al conjunto de ítems que constituyen la pregunta sobre discapacidad sugeridos por las Naciones Unidas para la ronda censal del 2010, haciendo menos viable la comparación de los datos (Reyes, 2014).

En el 2013, el Centro de Rehabilitación y Educación Especial (CREE) retomó el censo de PCD en el estado, en donde se realizó una cobertura de sólo 10 municipios (de los 106 que son) y donde manifestó que el porcentaje de PCD era de 1.93 por ciento, cifra menor comparada con la que ofrecen organismos como el INEGI de 6.4 por ciento (Díaz, 2013).

Los datos de la Encuesta Nacional de la Dinámica Demográfica en México (ENADID, 2014), revelan que, en la mayor parte del país, aún es amplia la brecha en la inserción de las PCD en el mundo del trabajo, lo cual se suma a múltiples desigualdades que se refuerzan entre sí.

Una vez presentado el panorama de la PCD en el mundo, el país y el estado, surge la necesidad de analizar por qué la inclusión laboral a PCD ha ido en decremento durante el periodo 2013-2018 en el sureste mexicano, a pesar de que en el país se cuenta con diferentes legislaciones, mecanismos y programas (nacionales y estatales) enfocados a la inclusión laboral a PCD. Ya que una de las grandes dificultades que presentan las PCD es que actualmente en el mundo existe una situación de discriminación, exclusión y estigmatización hacia este grupo. En el sureste mexicano no es la excepción, ya que su participación en el sector laboral es casi nula, como se pude apreciar al contrastar los datos estadísticos presentados anteriormente.

De igual forma es necesario aclarar que esta investigación se centró en la inclusión laboral de PCD que se realiza a través del programa de rehabilitación e inclusión laboral de PCD del CREE en el sureste mexicano, sin embargo, esto no significa que en el estado donde se realizó este estudio no se realice inclusión laboral a PCD a través de otros centros y/o 
instituciones.

\section{Marco teórico y referencial}

Durante la revisión de la literatura, se distinguen tres tipos de modelos que se refieren a la discapacidad. El primero en definir estos modelos es el autor Puig de la Bellacasa (1987), quien divide estos modelos como: el modelo tradicional, de rehabilitación y de la autonomía personal. Y de manera más reciente la autora Palacios (2008) los define como: el de prescindencia ${ }^{1}$ característico de la edad antigua y el medievo; el modelo médico o de rehabilitación, típico de la primera mitad del siglo XX y, por último, el modelo social, surgido a partir de la década de los sesenta del siglo pasado hasta hoy en día.

\section{Modelos según Palacios (2008)}

\section{Modelo de la Prescindencia}

Esta autora menciona que la actitud más común hacia la discapacidad en la antigüedad y la edad media era la prescindencia, ya sea por haber recibido un castigo de los dioses o bien por considerarse que las PCD no tenían nada que aportar a la comunidad, se asumía que sus vidas carecían de sentido y que, por lo tanto, no valía la pena que vivieran.

Este modelo se explica a partir de dos supuestos, uno relacionado con la causa de la discapacidad y otro con el rol del discapacitado en la sociedad. Respecto al primero propone que las causas que daban origen a la discapacidad eran religiosas, un castigo de los dioses por un pecado cometido generalmente por los padres de la persona con discapacidad, o bien una advertencia de la divinidad que, a través de una malformación congénita, podía estar anunciando que la alianza ancestral se había roto y que se avecinaba una catástrofe. En cuanto al segundo supuesto, que identificaba el rol de la persona con su utilidad, partía de la idea de que el discapacitado no tenía nada que aportar a la sociedad, que era un ser improductivo $\mathrm{y}$, por consiguiente, terminaba transformándose en una carga tanto para sus padres como para la misma comunidad.

\section{Modelo médico o rehabilitador}

A raíz de la Primera Guerra Mundial y de la introducción de las primeras legislaciones en torno a la seguridad social, el concepto de discapacidad asiste a un cambio de paradigma.

Fue así como el modelo de prescindencia pasó a ser sustituido por el modelo médico o de rehabilitación, cuyos fundamentos impregnan la mentalidad común hasta el día de hoy.

Los supuestos en los que se basa este nuevo paradigma son dos, uno relacionado con las causas de la discapacidad, y el otro con el rol de la persona en la sociedad: en primer término, las causas de la discapacidad ya no son religiosas sino científicas y, en segundo lugar, las PCD dejan de ser consideradas inútiles respecto de las necesidades de la comunidad y, siempre que sean rehabilitadas, pueden tener algo que aportar.

Palacios (2008) nos dice que con este modelo de rehabilitación emerge la posibilidad del trabajo protegido, pues los Estados comienzan a hacerse cargo de aquellos ciudadanos que poseen diversidades funcionales. Surgen así políticas públicas tendientes a poner los tratamientos médicos y los medios técnicos al servicio de las PCD.

En este contexto, la atención sanitaria adquiere un rol fundamental; y en la esfera política, la respuesta es la

\footnotetext{
${ }^{1}$ El modelo de prescindencia se caracteriza por entender que las causas que originan la discapacidad son religiosas y por considerar que las PCD son innecesarias, como consecuencia de esta visión la sociedad decide prescindir de las personas con discapacidad, a través de políticas eugenésicas, marginándolas o en el mejor de los casos, tratándolas como objeto de caridad (Palacios, 2008).
} 
adecuación de la atención sanitaria a las necesidades de las PCD. De ahí que, ligado a este modelo médico, tras la Primera Guerra Mundial, surgieran las leyes de servicios sociales para los veteranos de guerra con discapacidad y, en la década de los sesenta, dicha legislación de servicios sociales se extendiera a todas las PCD (Palacios, 2008).

\section{Modelo social}

En este modelo un lugar de entender la discapacidad como una carencia de la persona que se debe remediar en pro de la inserción se pasa a mirar las deficiencias como un producto social, resultado de las interacciones entre un individuo y un entorno no concebido para él. De este modo, el modelo social atenúa fuertemente los componentes médicos de la discapacidad y resalta los sociales.

Esta nueva visión de inclusión "desafía la verdadera noción de normalidad en la educación y en la sociedad sosteniendo que la normalidad no existe, sino que es una construcción impuesta sobre una realidad donde sólo existe la diferencia” (Palacios, 2008, p.129).

\section{Estigma y prejuicio}

El presente trabajo se basó en las teorías del estigma de Erving Goffman (1970) y de la naturaleza del prejuicio propuesta por Gordon Allport (1954), para comprender la situación que viven día con día las PCD durante su proceso de inclusión tanto social como laboral.

Goffman (1970) en su obra, Estigma: Identidad deteriorada, plasma una perspectiva de la relación del estigmatizado con los normales (personas sin estigma) y viceversa. En ella, se analizan los testimonios de personas con diferentes tipos de discapacidad y deficiencias (ciegos, parapléjicos, deformes, etc.).
Para Goffman (1970), la sociedad establece los medios para categorizar a las personas y el complemento de atributos que se perciben como corrientes y naturales en los miembros de cada una de esas categorías y es el medio social el que establece las categorías. Refiere que estigma es, una clase especial de relación entre atributo y estereotipo, el término estigma y sus sinónimos ocultan una doble perspectiva: el individuo estigmatizado, en la cual existe la incógnita de si su condición de diferente es desconocida o resulta evidente en el acto, o, por el contrario, no es conocida por quienes lo rodean, ante estas posibilidades surgen las situaciones del desacreditado y desacreditable.

Define tres tipos de estigmas, el primero de ellos son las abominaciones del cuerpo, las distintas deformidades físicas; luego, los defectos del carácter del individuo que se perciben como falta de voluntad, pasiones tiránicas o antinaturales, creencias rígidas y falsas, deshonestidad. Por último, los estigmas tribales de la raza, la nación y la religión, susceptibles de ser transmitidos por herencia y contaminar por igual a todos los miembros de una familia (Goffman, 1970).

Goffman (1970), asigna el nombre de normal a todos aquellos que no se apartan negativamente de las expectativas particulares que están en discusión, para estos, son bien conocidas las actitudes que adoptan hacia una persona que posee un estigma y las medidas que toman al respecto, ya que son precisamente estas respuestas las que la benevolente acción social intenta suavizar y mejorar. Se cree que la persona que tiene un estigma no es totalmente humana y partiendo de este supuesto se practica la discriminación, mediante la cual reducimos en la práctica, sin pensarlo, sus posibilidades de vida.

Parte del supuesto de que la teoría del estigma se construye para explicar la inferioridad y dar cuenta del 
peligro que representa esa persona, racionalizando a veces una animosidad que se basa en otras diferencias, como, por ejemplo, la de la clase social (Goffman, 1970).

Pasando a otro concepto fundamental que se presenta en las situaciones que viven las PCD en su paso por la inclusión, está el prejuicio. Allport (1954) nos explica que el prejuicio comúnmente se manifiesta en el trato con miembros individuales de grupos rechazados, tan común es este proceso que podríamos definir el prejuicio como: una actitud hostil o prevenida hacia una persona que pertenece a un grupo, simplemente porque pertenece a ese grupo, suponiéndose por lo tanto que posee las cualidades objetables atribuidas al grupo.

El prejuicio tiene dos ingredientes esenciales, tiene que haber una actitud favorable o desfavorable y debe estar vinculada a una creencia excesivamente generalizada (y por lo tanto errónea). Las declaraciones prejuiciosas expresan a veces el factor actitud, a veces el factor creencia (Allport, 1954).

\section{El centro de rehabilitación y educación especial (CREE) ${ }^{1}$ y el programa de rehabilitación e inclusión laboral a personas con discapacidad en el sureste mexicano}

Mediante el programa de atención a PCD del Sistema Nacional para el Desarrollo Integral de la Familia (DIF) se otorgan servicios dirigidos a la población que presenta algún tipo de discapacidad y que se encuentra en condiciones de vulnerabilidad social, mediante acciones de prevención, rehabilitación e integración social, a través de los centros estatales (Sistema Nacional DIF, 2016).
A lo largo del país, se cuenta al menos con un centro de rehabilitación por estado, el centro en el que nos enfocaremos tiene aproximadamente 40 años de existencia durante los cuales ha presentado modificaciones en su estructura, dado que las elecciones presidenciales del 2018 coincidieron con las elecciones gubernamentales, el personal de dicho centro y del programa, se encontraban en proceso de relevos y adaptación a la nueva dirección y personal durante el trabajo de campo de este proyecto.

A diferencia de otras organizaciones gubernamentales del sureste mexicano que también se relacionan con temas de inclusión y PCD, el CREE es el que tiene mayor injerencia y participación en el proceso de inclusión laboral de PCD por presentar un proceso más integral y ofrecer herramientas para apoyar a las empresas participantes o interesadas en participar en el programa y a los buscadores de empleo.

La inclusión laboral a PCD es un tema el cual el nuevo gobierno del país y del mismo estado han enfatizado desde que inició su toma de protesta, tanto el plan nacional de desarrollo (Presidencia de la República, 2019-2024) y el plan estatal de desarrollo manifiestan en sus líneas el apoyo a grupos en situación de vulnerabilidad (siendo las PCD uno de estos) como una prioridad en sus agendas.

\section{Metodología}

Todos los nombres que se mencionan en este trabajo han sido cambiados para mantener el anonimato de los participantes y de las unidades de análisis.

El trabajo que se presenta se realizó con un enfoque cualitativo a través del método de estudio de caso de

\footnotetext{
${ }^{1}$ El CREE es un programa concebido para proporcionar servicios de atención, prevención y rehabilitación a las personas con discapacidad temporal o permanente, su misión es reducir la incidencia de las discapacidades incrementando las acciones relacionadas con la prevención y hacer efectiva la rehabilitación integral mediante la vinculación de las acciones de salud con las de integración social (Gobierno de Yucatán, 2019).
} 
tipo interpretativo, apoyado con la teoría del Estigma de Goffman (1970) y la del prejuicio de Allport (1954) para poder corroborar los supuestos de esta teoría en el contexto estudiado. El objeto de estudio es analizar por qué la inclusión laboral a PCD ha ido en decremento durante el periodo 2013-2018 en el sureste mexicano.

La unidad de análisis fue el CREE ubicado en el sureste mexicano y los participantes, usuarios de este centro, personal que labora para el programa de inclusión laboral a PCD, responsable del departamento de fotocredencialización y empresarios que participaron y dejaron de participar en el programa durante el periodo 2013-2018. Para la obtención de la información las técnicas utilizadas fueron la observación participativa, revisión documental y entrevistas.

Cabe mencionar que anteriormente se había tenido la oportunidad de tener un acercamiento a dicho centro gubernamental, con al menos dos directores y un responsable diferentes a quienes actualmente se encuentran a cargo del centro y del programa de rehabilitación e inclusión laboral a PCD respectivamente.

Para el estudio de caso se utilizó la bitácora de campo, fotografías, grabaciones, todas ellas con consentimiento y conocimiento de los participantes, quienes se mostraron en todo momento accesibles.

A partir de la recopilación de información, se utilizó la técnica de análisis de contenido y triangulación de estos, con la información proporcionada durante las entrevistas $\mathrm{y}$ datos de registros que mantiene el programa de rehabilitación e inclusión laboral de PCD y otros departamentos relacionados con el programa de este CREE del sureste mexicano.

Con la finalidad de ilustrar las particularidades del caso, en la figura 1 se observa el diseño del caso de inclusión laboral a PCD en el sureste mexicano apoyándonos en la propuesta de Stake (2006). 


\section{Figura 1}

Diseño del caso de Inclusión Laboral a Personas con Discapacidad del Sureste Mexicano

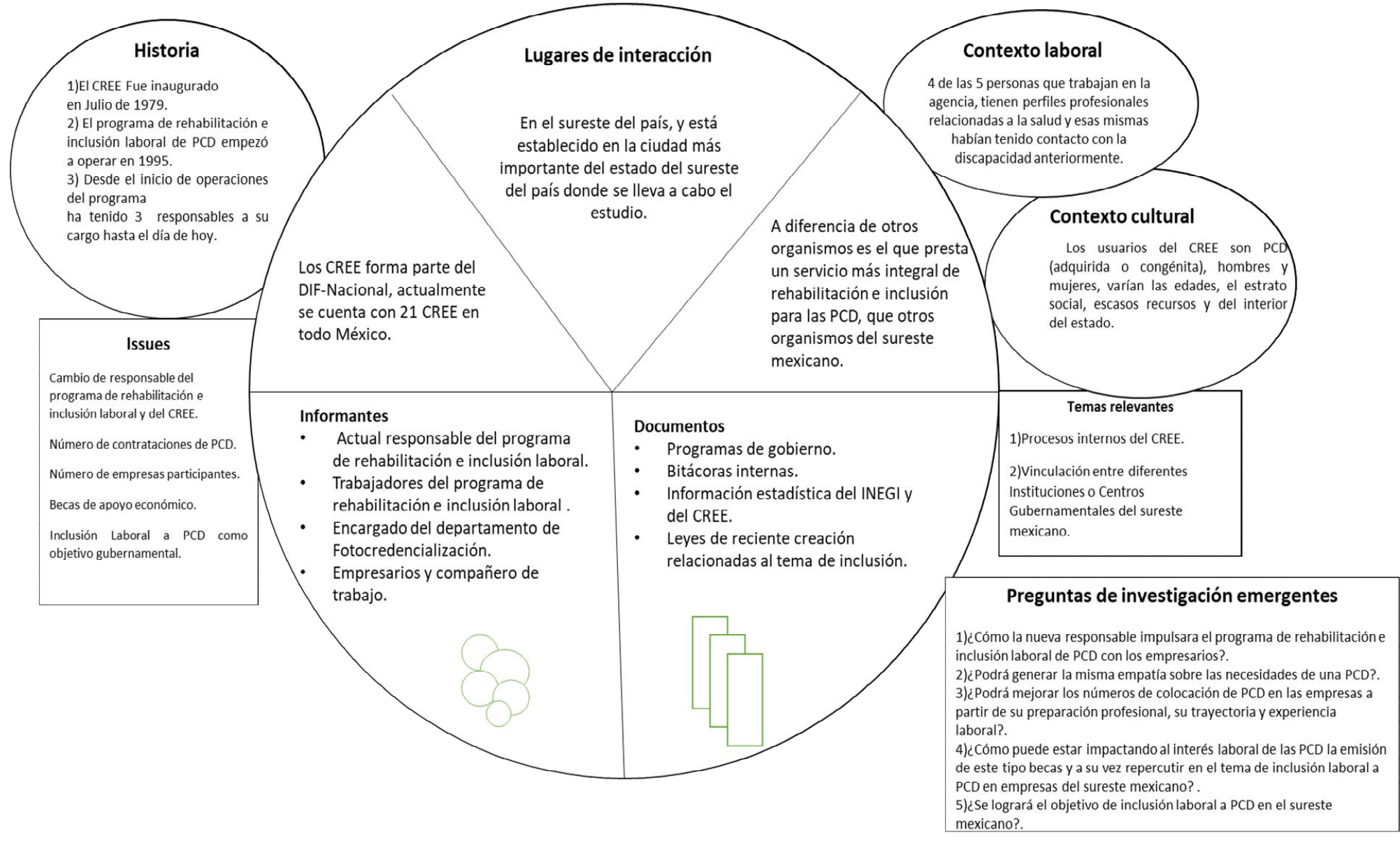

Fuente: Elaboración propia con base en Stake (2006). 
A partir de la recolección de datos a través de la observación, revisión documental y entrevistas, se llevó a cabo un análisis de datos a nivel textual, con el cual, se realizó un proceso de interpretación de los datos en una categorización de conceptos que mejor explicaban los fenómenos sociales y teóricos que ocurren alrededor del programa de rehabilitación e inclusión laboral donde se llevó a cabo el estudio. Las categorías y códigos que se emplearon para agrupar los datos que mejor explicaron el análisis textual fueron:

1. Categoría: Contacto mixto.

Códigos: cultura de contratación a PCD, temor, contacto con PCD.

2. Categoría: Objetivos hacia la inclusión.

Códigos: apoyo económico, asistencialismo, interés.

\section{Resultados}

\section{Inclusión laboral a personas con discapacidad ¿afinidad o estigma?}

“¡No, el no vino a pedir limosna, él está aquí trabajando!” (Miguel, responsable del departamento de fotocredencialización, diario de campo, 4 de octubre 2019).

En este proceso de cambios y relevos de personal del CREE y del programa, "Miguel" fue el anterior responsable de dicho programa y estuvo a cargo cerca de seis años. Es el tercero de siete hijos, desde la edad de ocho años empezó a trabajar en un puesto de periódicos junto a su padre, todos los días después de la escuela, su papá lo iba a buscar y ahí vendía periódicos junto con sus hermanos mayores.

Miguel tiene muy presente hasta el día de hoy, su experiencia en su primer día de trabajo, él estaba junto a su papá ayudándolo a cobrar, cuando de manera inesperada un señor se le acerca para regalarle unas monedas, y su papá de manera inmediata, le respondió a aquel señor: “¡no, el no vino a pedir limosna, él está aquí trabajando! ... y le devolvió el dinero”. Para él, recordar todo lo que su padre le enseñó le ha sido más útil que lo que aprendió en la escuela, ya que nunca hizo distinción entre sus hijos por la condición de Miguel, quien tiene discapacidad motriz.

Estudió hasta nivel de secundaria, posteriormente cursó estudios de analista programador de datos y desde muy temprana edad se vio involucrado en el ambiente laboral, en la política, en la organización de asociaciones civiles, y hasta en cuestiones legales, como participar en la sesión de la Ley para la Integración de PCD en el estado, finalmente después de muchos obstáculos, dicha ley fue aceptada y en una visita del presidente de la república de aquel entonces al estado, tuvo la oportunidad de coincidir con él, y le obsequia el escrito de la ley, y a partir de ese momento surge la invitación por parte del director del DIF de éste estado para que Miguel empiece a trabajar con ellos.

Pero fue hasta que llega al gobierno del estado una mujer al poder, que él se ve más involucrado en trabajar en una organización gubernamental relacionada con las PCD por parte del ayuntamiento y con el tiempo se dio la oportunidad de hacerse cargo de la agencia de integración laboral a PCD (llamada así durante esa administración al mismo programa que actualmente nos referimos como programa de rehabilitación e inclusión laboral para PCD).

Tuvimos buenos resultados de colocación, se dio una sinergia muy interesante cuando a mí me toca estar en la agencia, los amigos que se hicieron en el camino ya estaban en mejores posiciones y se pudo dar que nos 
facilitaran las cosas para colocar gente. (Miguel, responsable del departamento de fotocredencialización, diario de campo, 4 de octubre 2019).

Con los cambios en el CREE, su puesto también tuvo un cambio, en este momento es el responsable del departamento de fotocredencialización en el mismo centro de rehabilitación.

Actualmente el centro de rehabilitación a nombrado una nueva responsable del programa y también se ha renombrado como: "programa de rehabilitación e inclusión laboral de PCD”. Durante el periodo de observación, la nueva responsable: “Tatiana” tenía aproximadamente quince días de haber sido nombrada y de estar en su nuevo puesto.

En lo personal como rehabilitadora me ayudó porque pude entender lo que se esperaba de una persona con discapacidad, todo lo que es la fuerza, la resistencia, el equilibrio, la tolerancia, la frustración, el hacer una tarea tras otra, el permanecer de pie 8 horas, el comer rápido, el entrar a un cuarto frío, el trabajar con máquinas, todo eso, a mí me sirvió. (Tatiana, responsable del programa de rehabilitación e inclusión laboral de PCD, diario de campo, 23 de septiembre de 2019).

Tatiana, ya tiene once años formando parte del centro de rehabilitación, y surge su nombramiento como parte de los cambios en la estructura de dicho centro. Es licenciada en rehabilitación, madre de tres hijos, estuvo en el área clínica como responsable del programa del adulto mayor, y antes de su actual puesto, se le comisionó como entrenadora y capacitadora del centro de capacitación que se creó como convenio entre el centro de rehabilitación y una tienda de autoservicio, dicho centro de entrenamiento tiene sus instalaciones dentro del mismo CREE.

Para que Tatiana pudiera llegar a desarrollar el programa del centro de capacitación, tuvo que tomar un curso de aspirantes de nuevo ingreso de la tienda de autoservicio desde la parte administrativa hasta la operativa, como un aspirante más.

Fue una etapa de mi vida que marcó un cambio porque fue drástico, de estar coordinando un departamento de terapia física a pasar a ser una empleada de ocho horas, pero comprendí más la dignidad de un trabajo, al estar en contacto con otros aspirantes a ese puesto y ver como se valoran y colaboran para hacer equipo. (Tatiana, responsable del programa de rehabilitación e inclusión laboral de PCD, diario de campo, 23 de septiembre de 2019).

La importancia de este centro de entrenamiento es que prepara gente con discapacidad para formarse en un puesto operativo, que puede ser contratado por cualquier tienda de autoservicio de la zona con un giro similar a la cadena con la que se realizó el convenio. Hoy en día el centro de capacitación a graduado a 12 generaciones de PCD que han cursado y acreditado el curso, y desarrollado habilidades para poder ingresar a la vida laboral en puestos similares si así lo desearan.

Tatiana, desde su nuevo puesto, comenta que desea consolidar más este departamento, el equipo de trabajo con el que cuenta se mantiene igual a la administración pasada, menciona que es un equipo integrado y confía que con la experiencia con la que ya cuentan y el conocimiento de los procesos podrán tener más impacto, como agente de sensibilización para promover la cultura de la inclusión hacia las PCD. 
Ante este cambio de responsables del programa entre una administración y otra, surgen las siguientes cuestiones críticas: ¿Cómo la nueva responsable impulsará el programa de rehabilitación e inclusión laboral de PCD con los empresarios? ¿Podrá generar la misma empatía sobre las necesidades de una PCD? ¿Podrá mejorar los números de colocación de PCD en las empresas a partir de su preparación profesional, su trayectoria y experiencia laboral?
En la figura 2 se presenta la gráfica de personas colocadas en empresas participantes en el programa de rehabilitación e inclusión laboral y las empresas participantes en la administración pasada (2013-2018) y en la cual se puede apreciar el decremento de participación de las empresas y contrataciones a PCD durante el periodo estudiado.

\section{Figura 2}

Participación de Empresas en el Programa de Inclusión Laboral y Contratación de PCD

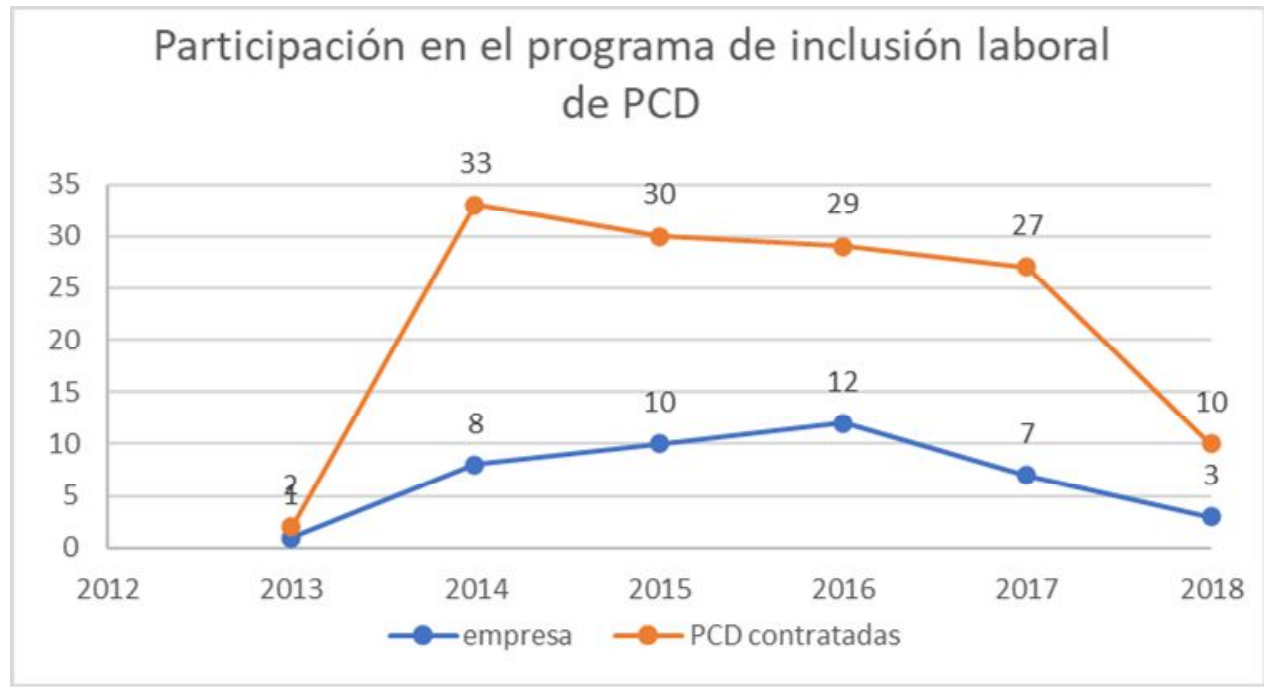

Fuente: Elaboración propia con información proporcionada por el CREE (2019), Yucatán.

La inclusión laboral a PCD ¿es realmente un objetivo para las agendas de gobierno federal de México y estatal del sureste mexicano?

“... desde que inició el año, hemos tenido una baja en las personas que han venido a solicitar trabajo” (Johana, trabajadora social, diario de campo, 19 de septiembre de 2019).

El programa de rehabilitación e inclusión laboral a
PCD, tiene sus propios requisitos, procesos y lineamientos y atiende a hombres y mujeres en edad laboral con cualquier tipo de discapacidad (auditiva, intelectual, neuromotora y visual).

Para poder participar en el programa los requisitos son:

- Credencial de discapacidad.

- Copia del Instituto Nacional Electoral (INE). 
- Copia actual de comprobante domiciliario.

- Copia de la clave única del registro de población (CURP).

De acuerdo con información obtenida del programa de rehabilitación e inclusión laboral a PCD (DIF-CREE, 2019) el proceso consta de los siguientes pasos:

1. Se hace un alta en el centro de rehabilitación estatal, donde se realizan diferentes evaluaciones médicas, psicológicas y funcionales.

2. La persona que acude por primera vez se refiere al área médica para la formación del expediente clínico en el centro de rehabilitación estatal. Posteriormente en el programa de rehabilitación e inclusión laboral empieza un estudio sociolaboral. Así mismo se aplican pruebas psicométricas y la del Sistema de evaluación de habilidades laborales diseñado para PCD y adultos mayores conocido como VALPAR $^{1}$ (Consejo para el Desarrollo y la Inclusión de las Personas con Discapacidad, 2017).

3. Los resultados de las pruebas determinan en qué tipo de trabajo se puede desempeñar la persona.

4. Se ofertan las vacantes pertinentes y se da inicio al proceso de inclusión laboral, lo cual va acompañado de una plática de sensibilización en las empresas.

5. Cuando la persona se encuentra en el ámbito laboral, se realiza un seguimiento de seis meses con el fin de obtener una permanencia laboral idónea.

Para que estos procesos se lleven a cabo, el programa cuenta con cinco personas trabajando de manera conjunta: una trabajadora social, una evaluadora de habilidades del Servicio Nacional del Empleo (SNE), una psicóloga, la secretaria de la persona responsable del programa y la responsable de dicho programa, la combinación de estas profesionales es para ofrecer un servicio más integral a las empresas y a los usuarios.

Durante el periodo de observación y entrevistas con el equipo de trabajo, dos personas coinciden en sus comentarios acerca de la afluencia de las PCD hacia el centro de rehabilitación y hacia el programa de rehabilitación e inclusión laboral de PCD.

“...en 6 meses más o menos, 5 meses se hicieron la cantidad de credenciales que se hacían en un año, como 3000 credenciales y se siguen haciendo todos los días de a 50 credenciales” (Johana, trabajadora social, bitácora de campo, 19 de septiembre de 2019).

...estamos viendo que, si hay un gran interés de la población, nosotros aquí lo vemos, por que acuden a buscar su credencial para PCD que aquí se emite, y si hemos visto una afluencia pues muy importante de personas que son de la ciudad y del interior de estado. (Tatiana, responsable del programa de rehabilitación e inclusión laboral de PCD, diario de campo, 23 de septiembre de 2019).

\footnotetext{
${ }^{1}$ En México, la STPS cuenta con Centros de Evaluación de Habilidades Laborales con el sistema VALPAR que son operados por el servicio Nacional de Empleo (SNE), con esta evaluación se identifican las necesidades, habilidades funcionales, aptitudes, logros e intereses de las personas. Actualmente operan 29 centros de habilidades laborales en 27 estados del país, estos centros funcionales en algunas oficinas del SNE o en alianza con instituciones públicas como el DIF (Consejo Nacional para el Desarrollo y la Inclusión de las Personas con Discapacidad, 2017).
} 
En relación con el tema de discapacidad, por mandato oficial, se creó un programa social denominado: Programa pensión para el bienestar de PCD, el cual arrancó la entrega de estos apoyos en enero del 2019, este programa apoya a niñas, niños y adolescentes, así como jóvenes ( 0 a 29 años) que tienen discapacidad permanente, y población indígena de 0 a 64 años. El programa otorga un apoyo económico a:

- Todas las PCD que pertenezcan a grupos indígenas del país de 0 a 64 años.

- Niñas, niños y jóvenes con discapacidad permanente de 0 a 29 años que vivan en municipios y zonas urbanas de alta y muy alta marginación.

- El monto de apoyo es de $\$ 2,550$ pesos (moneda nacional) que son entregados bimestralmente, la meta de población a atender son 1 millón de PCD.

El apoyo económico se entregará de manera directa, sin intermediarios, mediante el uso de una tarjeta bancaria (Secretaría del Bienestar, 2019).

“...en cierto sentido si consideramos que este tipo de apoyos, pueden representar un reforzamiento a la práctica asistencial” (Tatiana, responsable del programa de rehabilitación e inclusión laboral de PCD, diario de campo, 23 de septiembre de 2019).
Como se explicó, parte de los requisitos para poder empezar el proceso en el programa de rehabilitación e inclusión laboral de PCD, es contar con la credencial de discapacidad, en este caso Miguel en su nuevo puesto es él quién está encargado del departamento para realizar este primer requisito.

“... siento que, si va a perjudicar un poquito las ganas de salir delante de los compañeros con discapacidad, tal vez si hubiera mecanismos, aunque si, bueno este programa al menos tiene unos candados” (Miguel, responsable del departamento de fotocredencialización, diario de campo, 4 de octubre 2019).

Si bien es cierto que un objetivo prioritario y manifestado de la presidencia del país y del gobierno estatal es atender a grupos vulnerables, entre ellos a las PCD, las cuestiones críticas al respecto serían: ¿cómo puede estar impactando al interés laboral de las PCD la emisión de este tipo becas y a su vez repercutir en el tema de inclusión laboral a PCD en empresas del sureste mexicano? ¿realmente se logrará el objetivo de inclusión laboral a PCD en el sureste mexicano?

En la figura 3 y figura 4 se presentan los datos a los que hacen referencia el equipo de trabajo de la agencia de inclusión laboral, en relación con el interés y mayor afluencia de las PCD a registrarse para obtener su credencial y posteriormente solicitar su beca de apoyo económico, en un año de diferencia. 


\section{Figura 3}

Comparativo de Asistencia de PCD para Solicitar su Credencial

COMPARATIVO MESES AGOSTO, SEPTIEMBRE, OCTUBRE AÑOS
$\mathbf{2 0 1 8 / 2 0 1 9}$
\begin{tabular}{|c|c|c|c|}
\hline & & & \\
\hline MESES/GENERO & MUJERES & HOMBRES & TOTAL \\
\hline AGOSTO (2018) & 49 & 54 & 103 \\
\hline AGOSTO (2019) & 383 & 584 & 967 \\
\hline SEPTIEMBRE (2018) & 47 & 68 & 115 \\
\hline SEPTIEMBRE (2019) & 337 & 455 & 792 \\
\hline OCTUBRE (2018) & 68 & 97 & 165 \\
\hline OCTUBRE (2019) & 385 & 437 & 822 \\
\hline
\end{tabular}

Fuente: Elaboración e información proporcionada por el departamento de fotocredencialización del CREE, Yucatán (2019).

\section{Figura 4}

Gráfica Comparativa de la Asistencia de PCD para Solicitar su Credencial

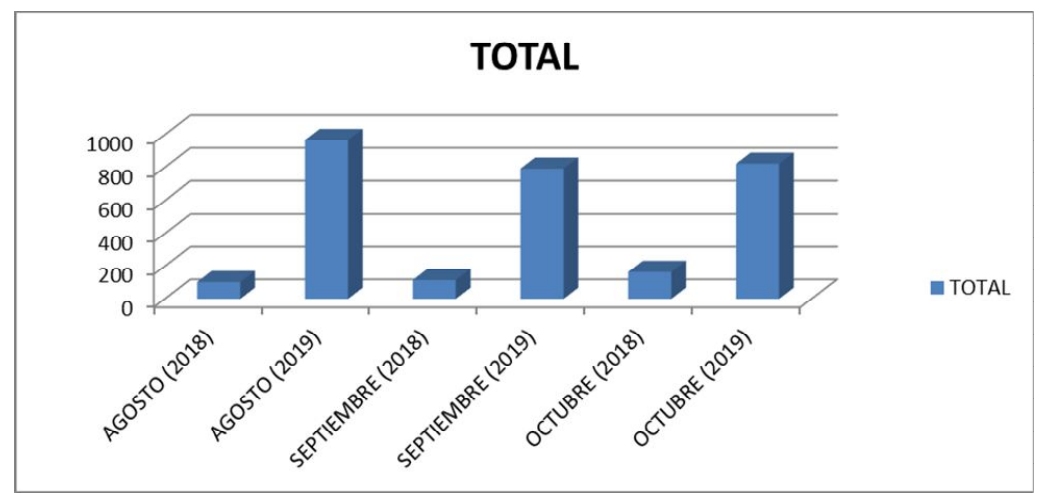

Fuente: Elaboración e información proporcionada por el departamento de foto credencialización del CREE, Yucatán (2019).

En cuanto a los usuarios del CREE, fue muy difícil conseguir entrevistas con ellos, sin embargo, se les realizaron preguntas referentes a los trámites que estaban haciendo o si conocían el programa de rehabilitación e inclusión laboral de PCD, de las 15 personas con las que se intentó tener contacto, 11 personas rechazaron la entrevista.

De los cuatro usuarios entrevistados, una madre y un menor de edad confirmaron que estaban en el CREE para solicitar su beca. Otro usuario, estaba en espera de su terapia y al preguntarle si conocía el programa de rehabilitación e inclusión laboral a PCD del CREE, comentó que no, pero que le gustaría tener la oportunidad de trabajar. Y, por último, al platicar con su tutor que lo acompañaba, mencionó que no conocía del programa, y que ya habían iniciado el trámite para solicitar el apoyo económico. 


\section{Experiencias del programa de rehabilitación e inclusión laboral de PCD en el sureste mexicano}

A continuación, se presenta la opinión del gerente regional que junto con su empresa participó de manera continua durante el periodo analizado y hasta el día de hoy siguen realizando contrataciones de PCD mediante el programa del CREE, para conocer su opinión acerca de la experiencia de participación.

...sin saber cómo opera el programa de rehabilitación e inclusión laboral de PCD del CREE, creo que un aspecto que podrían mejorar para fomentar la inclusión en el estado sería un tema de difusión hacia otras empresas, sus beneficios, etc. En el caso de nosotros fue un tema mutuo, pero a lo mejor hay empresas que no es que no quieran o ni siquiera saben del tema, pudieran tener cierta información con la difusión. (Alejandro, Gerente Regional del Sureste de tiendas de autoservicio, empresa participante en el programa, diario de campo, 01 de noviembre 2019).

El considera que parte de la idea de hacer inclusión de parte de su empresa es porque:

...tienen un enfoque muy humano, hay programas institucionales en la empresa relacionados a la inclusión laboral y de responsabilidad social. Es verdad que es mucho de la compañía, pero también es mucho de la gente con la que nos rodeamos acá. (Alejandro, Gerente Regional del Sureste de tiendas de autoservicio, empresa participante en el programa, diario de campo, 01 de noviembre 2019).
Con la finalidad de conocer la experiencia de los empresarios que dejaron de participar en el programa, se plasman fragmentos relacionados al tema de la inclusión laboral a PCD y su percepción hacia el programa.

...yo creo que al programa hay poco por hacer para mejorarlo, nosotros siempre tuvimos buena relación con el personal, en específico con la trabajadora social, lo único que sí nos gustaría como usuarios del servicio, que al final fue yo creo lo que apagó la flama, es que hay que trabajar con la bolsa de trabajo de las PCD, al principio tuvimos un resultado espectacular, entro más gente, cambió la situación, el personal del programa se dio cuenta de los focos rojos y las PCD ya no querían trabajar, nos daban excusas. Entonces nada más, si hubiera alguna manera de mantener esa chispa encendida de tener ganas de chambear, yo si recomendaría la inclusión laboral de PCD sin ningún problema, ellos nos cambiaron a nosotros, eso fue lo padre, aprendimos cosas de ellos y tuvimos que adaptar nuestros procesos. (Raúl, Gerente de producción, empresa que dejó de participar en el programa, diario de campo, 17 de octubre 2019).

... fue una buena experiencia, hubo un buen trato, quizá nos quedamos cortos en continuidad y seguimiento, entiendo que no todas las empresas estamos abiertas a hacer inclusión no por una cuestión de ideología sino a veces por políticas de la empresa y operación, entonces en ese sentido si te limita un poco. No recuerdo el haber sentido que tuve un servicio por parte de ellos (programa de rehabilitación), yo como 
cliente, siento que si pudo ser la falta de seguimiento, que podríamos estar haciendo algo más allá del resultado. (Sara, Gerencia de desarrollo humano, empresa que dejo de participar, diario de campo, 28 de octubre 2019).

Mi experiencia con la inclusión laboral a PCD fue grata, siempre conté con el apoyo del personal del programa, no tuve mayor complicación con el proceso, bueno hasta cierto punto, ya que la PCD que trabajo con nosotros se hacía a un lado, no la pudimos incluir la invitamos a comer, para comer todos juntos y ella se quedaba en su lugar y así, ella era muy cerrada, y es ahí donde tuvimos un poquito de problema, yo creo que deben hacerles estudios psicológicos a las PCD, yo creo que con la ayuda psicológica les asentaría bien tanto para ellos como para los contratantes. (Nadia, asistente de dirección, empresa que dejo de participar, diario de campo, 6 de noviembre 2019).

De primera instancia uno es temeroso a convivir con una PCD por ignorante, pero fue muy cómodo aprender a trabajar con una PCD y compartir todo. La naturaleza del proyecto de esta empresa es que necesito de un día para otro personal (PCD) y la respuesta del CREE ha sido un poco lenta, entre las recomendaciones hacia el programa sería el convocar empresarios para involucrar a PCD, hacer algún distintivo como gobierno para las empresas participantes o incentivo fiscal , que nos den datos de cuantas PCD hay en el estado, cuantos han sido evaluados, cuantos han sido contratados y así ... (Jacobo, Gerente Regional, empresa que dejo de participar, diario de campo, 6 de diciembre 2019).

Con estos fragmentos (los más significativos durante el trabajo de campo) podemos tener un panorama más completo de factores que pueden intervenir de manera favorable o no, en el proceso de inclusión. Desde sugerencias de mejoras en procesos internos del programa y del CREE, cuestiones mismas de las PCD, incentivos y difusión de parte del gobierno sobre temas de inclusión y de las instituciones que existen en el sureste para promover la inclusión laboral a PCD.

Es necesario proveer más información y sensibilización sobre el tema de discapacidad a la sociedad general, ya que se pudiese estar discriminando sin estar conscientes de esto. La evidencia de la falta de educación en estos temas en la sociedad mexicana se puede ver en los resultados publicados en la ENADIS (2005), donde se muestra que somos "una sociedad con intensas prácticas de exclusión, desprecio y discriminación hacia ciertos grupos" y "que la discriminación está fuertemente enraizada y asumida en la cultura social, y que se reproduce por medio de valores culturales” (ENADIS, 2010, p.6).

Para la CONAPRED (2012), en su reporte sobre la discriminación, consideran que el problema conceptual de origen sobre las PCD es que son vistas como una carga económica y no como sujetos de derechos. Las adecuaciones necesarias para su inclusión en el mundo laboral también son vistas como una carga adicional, en lugar de contabilizarse como un gasto normal y necesario, ya que siempre habrá clientes y potenciales empleados con discapacidad física o mental.

El mercado laboral mexicano cuenta con una demanda muy precaria de las personas con discapacidad. Se trata 
de una grave deficiencia que deja fuera del aparato productivo nacional a una fuerza laboral muy importante por el valor y talento que podrían agregar a la economía. Al mismo tiempo, arroja a una circunstancia de gran vulnerabilidad y dependencia a este sector de la población (CONAPRED, 2012).

\section{Conclusiones y Discusión}

Las empresas que se inscribieron para participar en el programa de inclusión laboral de PCD ha sido por medio de invitación directa del CREE o por que algún familiar con discapacidad ha acudido a terapias en dicho centro o por algún tipo de ayuda, sin embargo, desconocían el programa de rehabilitación e inclusión laboral de PCD de dicho centro, así como también los procesos que conlleva hacer inclusión laboral a PCD. Entendiendo que a este programa y al CREE les hace falta mayor promoción, para que más personas sepan cuáles son las funciones de este centro, sus alcances, los apoyos que brindan a las PCD y sus familias, entre capacitación, pláticas, talleres, etc.

El Programa Nacional de Trabajo y Capacitación para Personas con Discapacidad define inclusión laboral a PCD como: la inclusión de las y los trabajadores con discapacidad para trabajar en igualdad de condiciones que los demás, en un universo abierto donde la sociedad debe facilitar y flexibilizar las oportunidades en un ambiente propicio para el desarrollo emocional, físico e intelectual. Se entiende por inclusión laboral no solamente la incorporación de una persona con discapacidad para cubrir una vacante de acuerdo con su perfil y los requerimientos del puesto de trabajo. El término involucra desde la publicación de las vacantes, los procesos de selección y reclutamiento, la capacitación en y para el trabajo, la rehabilitación laboral, accesibilidad, seguridad y salud, igualdad en la remuneración y otras prestaciones, hasta los mecanismos para asegurar la estabilidad en el empleo, la promoción y el ascenso, entre otros (STPS, 2010-2012).

El CREE, a través del programa de rehabilitación e inclusión a PCD realiza una evaluación médicapsicológica de la PCD que sugiere los pasos hacia una integración de las PCD hacia las empresas, pero no hacia una inclusión laboral de la PCD al entorno laboral, ya que limita su campo de actuación hasta la primera parte de lo que se entiende por inclusión por la STPS (2010-2012), dejando fuera de su alcance los mecanismos para asegurar la estabilidad en el empleo, la promoción y el ascenso de la PCD.

El servicio que brinda el programa del CREE al empresario se queda corto en la capacidad de actuación del personal responsable del programa, ya que a pesar de que el número de empresas participantes en dicho programa es reducido, las funciones del personal se limitan a atender a las PCD durante su proceso de contratación pero sin dar seguimiento de su desempeño en la empresa, ya que se limita a hacer visitas a la empresa cuando se lo solicita su jefe inmediato o vuelve a tener contacto con la PCD cuando esta solicita específicamente algún apoyo.

Esta falta de seguimiento puede generar en los empresarios incertidumbre, confusión o desinterés, por la falta de orientación hacia ciertas situaciones que se pueden presentar en la empresa con los colaboradores que puedan no tener claro cómo interactuar con las PCD.

Un programa gubernamental como el observado, es un buen inicio hacia el camino de la inclusión, sin embargo, el programa por sí mismo no es la solución que requiere el tema de inclusión laboral. La inclusión laboral no sólo depende de los empresarios y de las PCD, o de las PCD y sus ganas de trabajar, sino depende de muchos actores más como: las autoridades, sociedad, sector empresarial, 
que permitan incluirlos no únicamente en el trabajo sino, también en el día a día. Todos estos actores forman parte del proceso y es indispensable su interés y acciones para que la inclusión laboral sea más que una realidad, una posibilidad.

Lo idóneo es que no existieran estos programas, que no fuera necesario recurrir a ellos, porque las personas, sin importar su condición, pudieran hacer valer sus derechos sin temor a ser rechazados, excluidos o estereotipados por las creencias que se han arrastrado a lo largo del tiempo, porque la sociedad debería evolucionar, cambiar sus paradigmas $\mathrm{y}$ ver las capacidades de las personas y no sus discapacidades. Aprender de lo que ha sucedido en relación con los casos de éxito empresariales con la inclusión laboral de PCD, para que sea a través de estos espacios laborales que las PCD puedan acceder a sus derechos y a la justicia social.

Se debe de resaltar la necesidad que tiene la sociedad de información y sensibilización en el tema de discapacidad, de la delgada línea que hay entre excluir y discriminar, la importancia de la educación en estos temas será una pieza clave al cambio de ideas erróneas sobre las capacidades de las PCD no sólo en el ámbito laboral.

Otra de las razones que pudo afectar a la inclusión laboral de PCD y el desempeño del programa, es el interés que presentan las PCD en solicitar la beca económica autorizada por el actual gobierno de la república a diferencia del interés que están presentando en registrarse para: solicitar trabajo, inscribirse a cursos de entrenamiento laboral que ofrece el CREE, dejar sus datos para la bolsa de trabajo del programa y/o registrarse para realizar sus pruebas de habilidades y psicológicas correspondientes al procedimiento. Al no haber interesados que pueda ofrecer el programa a las empresas interesadas en la inclusión laboral y con necesidad de cubrir una vacante laboral, el programa se queda sin su principal elemento para iniciar una vinculación hacia la inclusión laboral de PCD.

La disposición del apoyo económico, fue a partir de los últimos meses de la administración analizada, la cual ya venía en decremento de participación de empresas y contrataciones de PCD, por lo tanto, habría que considerar también entre los factores que pudieron dar origen a este comportamiento la opinión de los empresarios que participaron en el programa durante este periodo y dejaron de hacerlo o continuaron participando, de las PCD que han sido contratadas, así como también a las que se postularon y no fueron contratadas durante este periodo, la opinión de los compañeros de trabajo y los jefes directos de las PCD, para tener una comprensión más amplia sobre los diferentes actores del proceso de inclusión laboral.

En lo relacionado al objetivo de fomentar la inclusión desde la política nacional y gubernamental, pareciera acertado el hecho de fomentar el programa de apoyo económico para las PCD , sin embargo, no se menciona algún tipo de candado que permita hacer más eficiente la asignación de recursos por ejemplo: según el grado de afectación de la PCD, ya que hay ciertas discapacidades que dada su severidad, realmente les impedirá tener movilidad o permanecerán toda su vida postrados en una cama, por lo tanto la beca sería de mucho beneficio para ellos y sus familias, ya que la discapacidad es costosa (tratamientos, equipos, medicamentos, etc.) por el otro lado, hay discapacidades que su grado de afectación no es tan severa y les permite tener cierta movilidad y comunicación, en las cuales se sugeriría una canalización a programas de capacitación o entrenamiento laboral para que, después de sus pruebas, si son aptos a contratación, puedan participar en programas de inclusión laboral, ya que con estos apoyos económicos entregados sin mayor filtro, no se fomenta 
la inclusión, sino lo contrario: la exclusión (social y laboral) y el asistencialismo.

Otra cuestión que se debe considerar es el tiempo que estará vigente este apoyo, ya que queda claro que los recursos económicos son finitos, no alcanzará para todos los solicitantes, de hecho, está manifestado que sólo será para un millón de personas.

Ante el interés de las PCD por este apoyo económico, pudiera ser de mucha utilidad el acercamiento de las PCD hacia el centro de rehabilitación, ya que al tener mayor afluencia de PCD, se podrá tener censos más actualizados acerca de la cantidad de PCD que hay en este estado, en el interior del mismo y el tipo de discapacidad, cuestión que no se tiene actualizada ni completa a nivel estatal, ni a nivel nacional, ya que entre los censos del 2000 y del 2010 hay diferencias, debido a los cambios y omisiones al conjunto de ítems que constituyen la pregunta sobre discapacidad entre ambos censos, dificultando calcular la prevalencia de la discapacidad en los términos que se establecen por las naciones unidas, ya que no se sabe si las diferencias numéricas entre ambos censos, se deben a un cambio en el marco conceptual o a un cambio real en el volumen de PCD en el país (Reyes, 2014).

Con el comparativo de afluencia de PCD hacia la solicitud del apoyo económico, podemos darnos una idea de cómo será el comportamiento de la inclusión laboral de PCD los próximos años.

\section{Referencias}

Allport, G. (1954). La naturaleza del prejuicio. AddisonWesley Publishing Company, Inc.
Consejo Nacional para el Desarrollo y la Inclusión de las Personas con Discapacidad. (2017). Sistema VALPAR como método de evaluación, para la Inclusión laboral de las Personas con Discapacidad. Gobierno de México.

https://www.gob.mx/conadis/articulos/sistema-valparpara-la-inclusion-laboral-de-las-personas-condiscapacidad?idiom=es

Consejo Nacional para Prevenir la Discriminación (CONAPRED). (2012). Reporte sobre la discriminación en México 2012. CONAPRED. https://www.conapred.org.mx/documentos_cedoc/13\% 20\%20Reporte_2012_IntroGral_Ax.pdf

Díaz, C. (2013). Censan casa por casa a personas con capacidades diferentes (gráfico). Novedades Yucatán. https://sipse.com/novedades-yucatan/para-el-cree-ladiscapacidad-en-yucatan-es-menor-a-la-indicada-porel-inegi-64158.html

DIF-CREE. (2019). Programa de rehabilitación e inclusión laboral de personas con discapacidad (2019). Servicios, objetivo y nuestros procesos.

[Tríptico]Yucatán: Autor.

Encuesta Nacional de la Dinámica Demográfica (ENADID). (2014). Presentación de Resultados. INEGI.

http://www.beta.inegi.org.mx/proyectos/enchogares/es peciales/enadid/2014/

Encuesta Nacional sobre Discriminación (ENADIS). (2005). Primera Encuesta Nacional sobre Discriminación en México. CONAPRED, SEDESOL. https://www.conapred.org.mx/userfiles/files/Presentaci on_de_la_Encuesta_final.pdf 
Encuesta Nacional sobre Discriminación (ENADIS). (2010). Resultados Generales. CONAPRED. https://sindis.conapred.org.mx/investigaciones/encuest a-nacional-sobre-discriminacion-enadis-2010resultados-generales/

Gobierno Federal. (2010). Programa Nacional de trabajo y capacitación para personas con discapacidad 2010 2012. CONADIS.

http://www.stps.gob.mx/bp/secciones/transparencia/an exo/PCD_2010_2012.pdf

Secretaría del Bienestar. (2019). Programa pensión para el bienestar de las personas con discapacidad. Gobierno de México. https://www.gob.mx/bienestar/acciones-yprogramas/programa-pension-para-el-bienestar-de-laspersonas-con-discapacidad

Gobierno de Yucatán. (2019). DIF - Yucatán. Gobierno del Estado de Yucatán. http://dif.yucatan.gob.mx/creequienes-somos.html

Goffman, E. (1970). Estigma: La identidad deteriorada. Amorrortu

Instituto Nacional de Estadística y Geografía (INEGI). (2016). La discapacidad en México, datos al 2014. INEGI.

http://internet.contenidos.inegi.org.mx/contenidos/prod uctos/prod_serv/contenidos/espanol/bvinegi/productos/ nueva_estruc/702825090203.pdf

Organización para la Cooperación y el Desarrollo Económico (OCDE). (2010). Sickness, Disability and Work: breaking the barriers, a synthesis of findings across OECD countries. OECD. https://www.oecd.org/publications/sickness-disabilityand-work-breaking-the-barriers-9789264088856en.htm
Organización Mundial de la Salud (OMS). (2001). Clasificación internacional del funcionamiento (CIF), de la discapacidad y de la salud. OMS. https://www.who.int/disabilities/world_report/2011/su mmary_es.pdf

Organización Mundial de la Salud (OMS). (2011). Resumen informe mundial sobre la discapacidad. OMS.

https://www.who.int/disabilities/world_report/2011/es/.

Palacios, A. (2008). El modelo social de la discapacidad: orígenes, caracterización y plasmación en la convención Internacional sobre los Derechos de las Personas con Discapacidad. Cinca.

Puig de la Bellacasa, R. (1987). Concepciones, paradigmas y evolución de las mentalidades sobre la discapacidad. Discapacidad e Información, (14), 63-96. http://riberdis.cedd.net/bitstream/11181/3306/1/Discap acidad\%20e\%20informaci\%C3\%B3n_3ed.pdf

Presidencia de la República. (2019-2024). Plan Nacional de desarrollo 2019 - 2024 (PND). Presidencia de la República. https://lopezobrador.org.mx/wpcontent/uploads/2019/05/PLAN-NACIONAL-DEDESARROLLO-2019-2024.pdf

Reyes, J. (2014, agosto). La medición de la discapacidad en México en los censos de población 2000 y 2010: experiencia, resultados y retos. Ponencia presentada en el VI Congreso de la Asociación Latinoamericana de Población, Lima, Perú.

Secretaría de Trabajo y Previsión Social (STPS). (2010 2012). Programa Nacional de Trabajo y Capacitación para personas con discapacidad 2010-2012. Gobierno Federal. http://www.stps.gob.mx/bp/secciones/transparencia/an exo/PCD_2010_2012.pdf 
Sistema Nacional DIF. (2016). Agencias de Integración Laboral para personas con discapacidad. Gobierno de México. https://www.gob.mx/difnacional/acciones-yprogramas/agencias-de-integracion-laboral-parapersonas-con-discapacidad

Stake, R. (2006). Multiple case study analysis. The Guilford Press. 